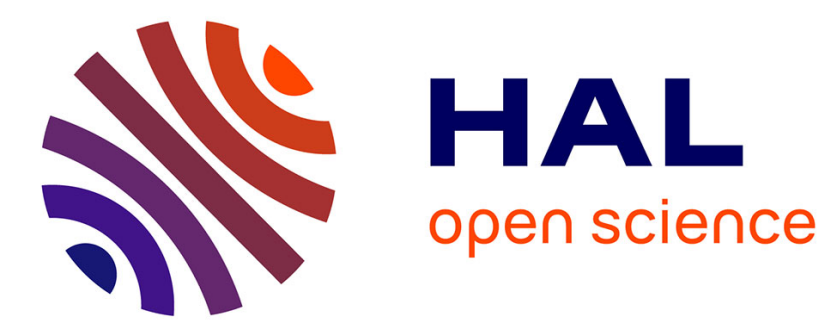

\title{
High expression of CPT1b in skeletal muscle in metabolically healthy older subjects
}

Cécile Bétry, E. Meugnier, M. Pflieger, G. Grenet, Serge Hercberg, Pilar

Galan, Emmanuelle Kesse-Guyot, Hubert Vidal, M. Laville

\section{To cite this version:}

Cécile Bétry, E. Meugnier, M. Pflieger, G. Grenet, Serge Hercberg, et al.. High expression of CPT1b in skeletal muscle in metabolically healthy older subjects. Journal of Diabetes \& Metabolism, 2019, 45 (2), pp.152-159. 10.1016/j.diabet.2018.01.018 . hal-01981585

\section{HAL Id: hal-01981585 \\ https://hal.science/hal-01981585}

Submitted on 22 Oct 2021

HAL is a multi-disciplinary open access archive for the deposit and dissemination of scientific research documents, whether they are published or not. The documents may come from teaching and research institutions in France or abroad, or from public or private research centers.
L'archive ouverte pluridisciplinaire HAL, est destinée au dépôt et à la diffusion de documents scientifiques de niveau recherche, publiés ou non, émanant des établissements d'enseignement et de recherche français ou étrangers, des laboratoires publics ou privés.

\section{(ㄷ)(1) $\$$}

Distributed under a Creative Commons Attribution - NonCommerciall 4.0 International 


\section{High expression of $C P T 1 b$ in skeletal muscle in metabolically healthy older subjects}

Cécile Bétry ${ }^{\mathrm{a}, \mathrm{b}}$, Emmanuelle Meugnier ${ }^{\mathrm{a}, \mathrm{b}}$, Marie Pflieger ${ }^{\mathrm{a}, \mathrm{b}}$, Guillaume Grenet $^{\mathrm{b}}$, Serge

Hercberg $^{\mathrm{c}, \mathrm{d}}$, Pilar Galan ${ }^{\mathrm{c}}$, Emmanuelle Kesse-Guyot $^{\mathrm{c}}$, Hubert Vidala, ${ }^{\mathrm{a}, \mathrm{b}}$, Martine Laville ${ }^{\mathrm{a}, \mathrm{b}}$

a Université de Lyon, Laboratoire CarMeN, INSERM U1060, INRA U1397, Université Claude Bernard Lyon

1, INSA-Lyon, Faculté de médecine Lyon-Sud, Oullins, F-69600, France

${ }^{\mathrm{b}}$ CRNH-RA, Hospices Civils de Lyon, Lyon, France

${ }^{\mathrm{c}}$ Université Paris 13, Equipe de Recherche en Epidémiologie Nutritionnelle (EREN), Centre de Recherche en Epidémiologie et Statistiques Sorbonne Paris Cité, Inserm (U1153), Inra (U1125), Cnam, COMUE Sorbonne Paris Cité, F-93017 Bobigny, France

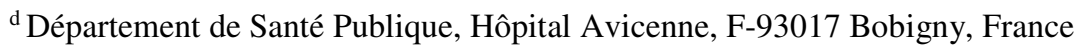

\section{Corresponding author:}

\section{Cécile Bétry}

David Greenfield Human Physiology Unit

The University of Nottingham Medical School, Queen's Medical Centre

Nottingham NG7 2UH, United Kingdom

Tel +33655956599

Email: cecile.betry@yahoo.fr

Received 23 July 2017; Accepted 14 January 2018

${ }^{1}$ Present affiliation: David Greenfield Human Physiology Unit, The University of Nottingham, Medical School, Nottingham, UK 


\begin{abstract}
Abbreviations
Acetyl-CoA acyltransferase 2, ACAA2; acetyl-CoA carboxylase beta, ACACB; body mass index, BMI; carnitine palmitoyltransferase $1 \mathrm{~b}, \mathrm{CPT} 1 \mathrm{~b}$; carnitine palmitoyltransferase 2 , CPT2; Center for Epidemiologic Studies Depression Scale, CES-D; Cognitive Difficulties Scale, CDS; fat-free mass index, FFMI; glycogen synthase 1, GYS1; hypoxanthine phosphoribosyltransferase 1, HPRT1; malonyl-CoA decarboxylase, MLYCD; Mini-Mental State Examination, MMSE; messenger RNA, mRNA; PGC-1-alpha, PPARGC1A; polymerase chain reaction, PCR; respiratory quotient, RQ; resting energy expenditure, REE; sirtuin, SIRT; standard deviation, SD; standard error of mean, SEM; Supplémentation en Vitamines et Minéraux Antioxydants, SU.VI.MAX.
\end{abstract}




\begin{abstract}
Aim. - Ageing is often associated with metabolic abnormalities such as insulin resistance, although some people remain metabolically healthy throughout their lives. The aim of this study was to gain more insight into metabolic health with increasing age.

Methods. - Two groups of robust and of frail subjects, respectively, were identified based on a composite ageing indicator and recruited from the French SU.VI.MAX 2 cohort of older disease-free subjects. In all, 14 men and 12 women, aged $67 \pm 4$ years, with similar anthropometric and metabolic characteristics at baseline (BMI: $24.5 \pm 2.9 \mathrm{~kg} \cdot \mathrm{m}^{-2}$ ) were included in the COMPALICLAMP study. Skeletal muscle biopsy was performed to assess expression of a set of metabolic and sirtuin (SIRT) genes. Also, whole-body substrate oxidation and insulin sensitivity were determined using the euglycaemic-hyperinsulinaemic clamp and indirect calorimetry techniques.

Results. - Robust subjects were more insulin-sensitive, oxidized more lipid in a fasting state and stored more glucose during the euglycaemic-hyperinsulinaemic clamp than did frail subjects. At the gene-expression level in skeletal muscle, carnitine palmitoyltransferase $1 \mathrm{~b}$ (CPT1b) messenger RNA (mRNA) levels were around four times higher in the robust compared with frail counterparts. Moreover, both SIRT2 and SIRT6 expression was lower in robust subjects and correlated with $C P T 1 b$ expression.

Conclusion. - CPT1b overexpression could be helping to maintain metabolic health with increasing age. Thus, it is suggested that targeting CPTIb expression might be an interesting strategy to counteract frailty at an early stage. In addition, future studies should examine the role of sirtuin in $C P T 1 b$ expression regulation.
\end{abstract}


Keywords: Ageing; Calorimetry; Glucose clamp technique; Insulin resistance; Sirtuins 


\section{Introduction}

Ageing is a complex multifactorial and continuous process [1]. Given the dramatic increase in average lifespan, healthy ageing and autonomy are now regarded as both a personal and a public health challenge. To that extent, frailty, which may be a reversible step before loss of autonomy, has generated much research interest [2]. Frailty is defined as an age-related condition with reduced functional reserves. It is intertwined with disability, sarcopenia and various disorders, including metabolic diseases like type 2 diabetes mellitus (T2DM) [3]. On the other hand, some robust people maintain good metabolic health throughout their lives, thereby highlighting the diversity of phenotypes of ageing [4].

In recent years, a large bulk of research has focused on skeletal muscle ageing. Growing old is not only associated with loss of skeletal muscle mass, but also with functional alterations in skeletal muscle such as insulin resistance, and both are involved in the onset of frailty [5]. Nevertheless, most of our knowledge of skeletal muscle and ageing-for example, development of insulin resistance, reduced fat-free mass, reduced mitochondrial capacity and quantity, and increased intramyocellular lipid contents-results from studies comparing older and younger subjects [6,7]. Thus, important differences in the way every individual faces growing old are rarely considered.

With the Implication of Metabolic and Genomic Modifications in Elderly Subjects (COMPALICLAMP) study, the aim was to determine the metabolic characteristics associated with frailty and robustness in healthy older subjects. For this purpose, wholebody substrate oxidation and insulin sensitivity were assessed to provide new insights at a molecular level into the expression of a set of candidate genes in skeletal muscle. Subjects 
were recruited from the French cohort of the Supplémentation en Vitamines et Minéraux Antioxydants 2 (SU.VI.MAX 2) Study.

\section{Materials and methods}

\section{SU.VI.MAX and SU.VI.MAX 2}

These two studies have been well described in previous reports [8,9]. In brief, all participants in the SU.VI.MAX 2 Study were invited to undergo a medical checkup, including an overall clinical examination. They also underwent the Mini-Mental State Examination (MMSE) and Dubois five-word test (5WT) for assessment of cognitive function, as well as the self-reported Center for Epidemiologic Studies Depression Scale (CES-D) and Cognitive Difficulties Scale (CDS). Furthermore, a muscle performance evaluation was carried out, including the Timed Up \& Go (TUG) Test, Chair Stand Test, and tests of walking speed and of balance over a 30-sec interval. A score ranging from 0 , the worst, to 3 , the best, was subsequently assigned to each test/scale. From this assessment, a composite ageing indicator was computed as the sum of the quartile of the six tests—namely, the MMSE, 5WT, TUG Test, Chair Stand Test, walking speed and balance tests, and CES-D (reversed) and CDS. Thus, their potential ageing indicator scores ranged from 0 to 24. Participants were grouped into tertiles according to this ageing indicator: those in the first tertile were assigned to the frail group, while those in the third tertile were assigned to the robust group.

\section{The COMPALICLAMP study}


From among the SU.VI.MAX 2 study participants, subjects from both the robust and frail groups were recruited, in the Lyon region (France), to take part in the COMPALICLAMP study. Inclusion criteria were age $>60$ years, body mass index (BMI) score $\geq 18 \mathrm{~kg} \cdot \mathrm{m}^{-2}$ but $<35 \mathrm{~kg} \cdot \mathrm{m}^{-2}$ and stable weight for the past 3 months. Participants were also non-smokers, and free of diabetes and digestive, cardiovascular, renal, liver or tumour diseases. Ultimately, 26 subjects (15 frail, 11 robust) were included (see ‘Subject inclusion' below).

The COMPALICLAMP study was conducted according to Declaration of Helsinki guidelines and approved by the local ethics committee. Written informed consent was obtained from all subjects. The study was registered at clinicaltrials.gov as NCT00951392.

\section{Study design}

After the inclusion visit, each participant attended the Research Centre for one day of comprehensive metabolic exploration after a 10-h overnight fast. All subjects underwent percutaneous biopsy of the vastus lateralis muscle, as previously described [12]. This skeletal muscle tissue $(62 \pm 28 \mathrm{mg}$ wet weight) was taken under local anaesthesia $(2 \%$ lidocaine) and immediately frozen in liquid nitrogen, then stored at $-80^{\circ} \mathrm{C}$. The biopsy was performed before the clamp study to avoid the confounding effect of insulin infusion on gene expression.

\section{Euglycaemic-hyperinsulinaemic clamp}

Cannulae were inserted into both antecubital veins for blood sampling, and for glucose and insulin administration. After a 1-h baseline period, the euglycaemic- 
hyperinsulinaemic clamp was performed for $2 \mathrm{~h}$. Exogenous insulin was administered as a primed continuous infusion at a rate of $1 \mathrm{mIU} \cdot \mathrm{kg}^{-1} \cdot \mathrm{min}^{-1}$, while the variable glucose infusion rate was adjusted to maintain blood glucose concentration at the target level of 5.5 $\pm 0.5 \mathrm{mmol} / \mathrm{L}$. Venous blood samples were collected every $10 \mathrm{~min}$ for measurement of glucose concentration using the hexokinase method (Olympus analyzer kit; bioMérieux SA, Marcy l'Etoile, France), and also at seven time points (T-20, T-10, T0, T90, T100, T110, T120 min) for measurement of plasma insulin (BI-INS immunoradiometric assay kit; CIS Bio International, Gif-sur-Yvette, France) and fatty acids (NEFA-C kit, Wako Chemicals, Neuss, Germany). The M value was determined during the final 30 min of the 2-h clamp.

\section{Indirect calorimetry}

These measurements were taken with a Deltatrac metabolic monitor (DatexOhmeda Oy, Helsinki, Finland) during the basal period (T-40 to T0 min) and at the end of the clamp (T60 to T120 min). Oxygen consumption $\left(\mathrm{VO}_{2}\right.$ in $\mathrm{L} / \mathrm{min}$ ) and carbon dioxide production $\left(\mathrm{VCO}_{2}\right.$ in $\left.\mathrm{L} / \mathrm{min}\right)$ were recorded every minute. Lipid and carbohydrate oxidation rates and resting metabolic rates were calculated using Ferrannini's equation [13]. Respiratory quotient (RQ) was assessed as the $\mathrm{VCO}_{2} / \mathrm{VO}_{2}$ ratio. To calculate protein oxidation, urine samples were collected twice a day (at T0 and T120 min) for measurement of urinary nitrogen using a chemiluminescence method (Antek 7000; Alytek, Juvisy, France).

\section{Body composition}


This was determined using a bioelectrical multifrequency bioimpedance analyzer (Spar 50, Spengler SAS, Aix-en-Provence, France). The fat-free mass index (FFMI) was calculated by dividing fat-free mass (in $\mathrm{kg}$ ) by height (in $\mathrm{m}$ ) squared.

mRNA expression

In skeletal muscle

Tissue samples were pulverized in liquid nitrogen, and total RNA was prepared using the mirVana miRNA Isolation Kit (Thermo Fisher Scientific, Waltham, MA, USA), according to the manufacturer's instructions. RNA concentrations were analyzed using a NanoDrop ND-1000 spectrophotometer (Thermo Fisher Scientific). mRNA levels were measured by reverse transcription followed by real-time polymerase chain reaction (PCR). Reverse transcription was performed with $500 \mathrm{ng}$ of total RNA using SuperScript II and RNAse H treatment (Thermo Fisher Scientific), as previously described [14]. Real-time PCR was performed with a Rotor-Gene 6000 cycler (QIAGEN N.V., Venlo, The Netherlands) on a final volume of $20 \mu \mathrm{L}$, containing $5 \mu \mathrm{L}$ of a 60 -fold dilution of synthesized complementary DNA (cDNA) and $15 \mu \mathrm{L}$ of reaction buffer (ABsolute Blue QPCR Mix, SYBR Green Plus ROX; Thermo Fisher Scientific), and 10.5 pmol of forward and reverse primers [14]. A standard curve was systematically generated with eight different amounts of purified cloned cDNA, and each assay was performed in duplicate. Values were normalized against hypoxanthine phosphoribosyltransferase 1 (HPRT1), a reference gene that is similar across samples. 
Based on the obtained results for lipid oxidation and whole-body insulin action (see 'Results' below), and the available data in the literature regarding alterations in skeletal muscle with increasing age $[15,16]$, our genomic study focused on the expression of a subset of genes encoding key enzymes of lipid metabolism [CPT1B and $C P T 2$, acetylcoenzyme A (CoA) acyltransferase $2(A C A A 2)$, acetyl-CoA carboxylase beta $(A C A C B)$, malonyl-CoA decarboxylase $(M L Y C D)$ ] and glucose metabolism [glycogen synthase 1 $(G Y S 1)$ ], and a key regulator of lipid oxidation [peroxisome proliferator-activated receptorgamma coactivator 1-alpha (PPARGC1A) and pyruvate dehydrogenase kinase 4 (PDK4)]. In addition, the expression of seven sirtuin genes (SIRT1-SIRT7) were also investigated, as these sirtuin proteins have been demonstrated to be major actors in the metabolism of ageing [17]. The primer sequences used are listed in Table S1 (see supplementary materials associated with this article online).

\section{In peripheral blood leucocytes}

Fresh blood samples were collected in PAXgene Blood RNA System tubes (PreAnalytiX, QIAGEN) and stored at $-80^{\circ} \mathrm{C}$ until needed for processing. Total RNA was isolated using PAXgene Blood RNA Kits (QIAGEN) as per manufacturer's instructions: $500 \mathrm{ng}$ of total RNA underwent reverse transcription, followed by real-time PCR as described above.

\section{Statistical analyses}


Values are presented as either means \pm standard deviation (SD) when pertaining to the entire cohort or as means \pm standard error of mean (SEM) after adjustment by group (frail or robust). Variables were considered to not be normally distributed using the Shapiro-Wilk test [18]. On inclusion, comparisons between the robust and frail groups were performed using Student's $t$ test and the Wilcoxon non-parametric test when necessary. Fisher's exact test for categorical variables was applied to compare gender ratios. Similar statistical analyses were also performed to compare the biopsy group with the entire group.

In addition, given the difference in age between the two groups, subsequent comparisons were also assessed by multiple linear regression analyses adjusted for age. For each analysis, the residuals were normally distributed. Dixon's Q test was used to exclude single outliers in the mRNA analysis. Also, as CPTIb mRNA levels were not normally distributed, correlations between $C P T I b$ and SIRT mRNA levels were determined by Spearman's correlation coefficients (rho) with bootstrap 95\% confidence intervals (CIs). A value of $P<0.05$ was considered significant.

All statistical analyses and plots were performed using RStudio 1.0.136 software [19]. The RVAideMemoire package with the 'spearman.ci' function was used for the bootstrap CIs, and the outliers package with the 'dixon.test' function for excluding an outlier. Scatterplots were fitted with the 'scatter.smooth' function.

\section{Results}

\section{Subject inclusion}


A flow chart of participants' inclusion into our study is shown in Fig. 1. One of the eight frail subjects was subsequently excluded from mRNA analysis because the mRNA levels of SIRT1, SIRT2 and SIRT3 were all outliers $(P<0.001, P<0.05$ and $P<0.001$, respectively, by Dixon's Q test; Fig. S1; see supplementary materials associated with this article online). Nevertheless, significance was not altered when the analysis was run with and without this subject (data not shown). Furthermore, anthropometric and metabolic characteristics at the time of screening did not differ between the biopsy group $(n=16)$ and the entire group ( $n=26$; Table S2; see supplementary materials associated with this article online).

\section{Subject characteristics}

Participants' characteristics when screening for frail and robust subjects are presented in Table I. Their average age was $67 \pm 4$ years, with a gender ratio of 14 men to 12 women and a mean BMI of $24.5 \pm 2.9 \mathrm{~kg} \cdot \mathrm{m}^{-2}$. As expected with subjects free of diabetes, their mean fasting glycaemia was $5.3 \pm 0.5 \mathrm{mmol} / \mathrm{L}$ and mean $\mathrm{HbA} 1 \mathrm{c}$ was $5.6 \pm 0.3 \%$. Given their inclusion on the basis of the ageing indicator, this was significantly lower in the frail compared with the robust group. Furthermore, frail subjects were 5 years older than robust subjects $(P<0.01)$; given this difference, all subsequent comparisons were adjusted for age. Otherwise, the general anthropometric and metabolic characteristics were similar between both groups.

\section{Body composition}


There were no differences in terms of body composition between the two groups, with a fat-free mass of $51.1 \pm 2.4 \mathrm{~kg} v s 53.0 \pm 3.3 \mathrm{~kg}$, and a fat mass of $15.3 \pm 1.0 \mathrm{~kg} v s$ $16.1 \pm 1.3 \mathrm{~kg}$, for frail $v s$ robust subjects, respectively (all $P>0.6$ ). FFMI was also similar (frail: $18.5 \pm 0.5 \mathrm{~kg} \cdot \mathrm{m}^{-2}$; robust: $19.1 \pm 1.0 \mathrm{~kg} \cdot \mathrm{m}^{-2} ; P>0.6$ ).

\section{Whole-body substrate oxidation}

As shown in Table II, resting energy expenditure (REE) did not differ between frail and robust subjects. Both groups displayed significant differences in whole-body substrate oxidation at baseline, with markedly higher lipid oxidation in robust subjects $(P<0.05)$. As a result, the RQ tended to be lower in that group $(P=0.07)$. However, during the euglycaemic-hyperinsulinaemic clamp, no differences were found, although robust subjects displayed significantly higher non-oxidative glucose metabolism $(P<0.01)$.

\section{Insulin sensitivity}

During the euglycaemic-hyperinsulinaemic clamp, no differences were found in glycaemia (frail: $6.1 \pm 0.2 \mathrm{mmol} / \mathrm{L} v s$ robust: $6.0 \pm 0.2 \mathrm{mmol} / \mathrm{L}$ ), insulinaemia (frail: $37.0 \pm$ $2.6 \mathrm{mIU} / \mathrm{L} v s$ robust: $37.5 \pm 3.4 \mathrm{mIU} / \mathrm{L}$ ), fatty acids (frail: $39 \pm 6 \mu \mathrm{mol} / \mathrm{L} v s$ robust: $30 \pm 6$ $\mu \mathrm{mol} / \mathrm{L}$ ) or fatty acid suppression (frail: $92.1 \pm 1.1 \%$ vs robust $93.7 \pm 1.4 \% ; P>0.35$ for all). The $M$ value, an index of insulin sensitivity, was significantly higher in robust subjects (frail: $7.4 \pm 0.6 \mathrm{mg} \cdot \mathrm{kg}^{-1} \cdot \mathrm{min}^{-1} v s$ robust: $8.2 \pm 0.8 \mathrm{mg} \cdot \mathrm{kg}^{-1} \cdot \mathrm{min}^{-1} ; P<0.01$ ) even after normalization for fat-free mass (frail: $10.0 \pm 0.7 \mathrm{mg} \cdot \mathrm{kg}^{-1} \cdot \mathrm{min}^{-1} \mathrm{vs}$ robust: $10.9 \pm 1.2 \mathrm{mg} \cdot \mathrm{kg}^{-}$ $\left.{ }^{1} . \min ^{-1} ; P<0.05\right)$. 


\section{Expression of metabolic genes in skeletal muscle}

The mRNA levels of eight genes involved in skeletal muscle lipid and glucose metabolism are presented in Fig. 2. The level of $C P T 1 b$ mRNA was around four times higher in robust subjects $(P<0.05)$. Moreover, it correlated with the ageing indicator (rho: 0.734, 95\% CI: $0.343-0.881 ; P<0.01)$. Otherwise, mRNA levels did not significantly differ between the two groups.

\section{Expression of sirtuin genes}

Skeletal muscle expression levels of the seven SIRT genes (SIRT1-SIRT7) are reported in Fig. 3 (except for SIRT4, which was undetectable); SIRT2 and SIRT6 mRNA levels were lower in robust than in frail subjects $(P<0.05$ and $P<0.01$, respectively). Sirtuin gene expression was also measured in peripheral blood leucocytes to potentially determine circulating biomarkers able to discriminate robust from frail subjects. However, SIRT1, SIRT2 and SIRT6 mRNA levels did not differ between the two groups when measured in peripheral blood leucocytes (Table S3; see supplementary materials associated with this article online).

\section{Correlation between CPT1b and sirtuin gene expression}

As revealed in Fig. 4, mRNA expression levels of $C P T 1 b$ strongly correlated with SIRT1 (rho: $0.675,95 \%$ CI: $0.122-0.935 ; P<0.01$ ) and SIRT6 (rho: -0.814 ; 95\% CI: $0.936,-0.512 ; P<0.001)$ in skeletal muscle. Using multiple linear regression analysis with 
$C P T 1 b$ as the dependent variable, and SIRT1 or SIRT6, age, gender and BMI as independent variables, both SIRT1 and SIRT6 remained significantly correlated $(P<0.01$ and $P<0.05$, respectively; data not shown).

\section{Discussion}

With increasing age, age-related changes in skeletal muscle mass and function are at least partly responsible for a wide range of disabilities and metabolic diseases, including T2DM. Until now, physiological and metabolic studies in the field of skeletal muscle ageing have largely been based on comparisons between older and younger subjects. However, phenotypes in older individuals are heterogeneous, and a better understanding of the metabolic differences among older people may help to identify relevant targets for promoting healthy metabolic ageing.

Our present study compared robust and frail, but otherwise disease-free, older individuals to better understand the potential factors involved in metabolic health with older age. The most striking result to emerge from our data was that robust subjects had a fourfold higher level of $C P T 1 b$ mRNA than did frail subjects. In good agreement with such overexpression, those subjects also oxidized more lipid in a fasting state. These findings support preclinical studies in which experimental overexpression of $C P T 1 b$ enhanced fatty acid oxidation and, more importantly, partially reversed the skeletal muscle alterations associated with ageing [20,21]. It may also be assumed that the overexpression of $C P T 1 b$ accounts for the greater insulin sensitivity of robust subjects due to their lower intramyocellular lipid contents. This hypothesis is consistent with a previous clinical study 
showing that levels of metabolites derived from intramyocellular lipids is the same in older insulin-sensitive and younger subjects, whereas it is higher in older insulin-resistant subjects [22]. Moreover, in that same study, levels of $C P T 1 b$ mRNA transcripts were higher, albeit not significantly so, in older insulin-sensitive subjects than in older insulinresistant subjects [22]. In the present study during the euglycaemic-hyperinsulinaemic clamp, our robust subjects also displayed higher levels of non-oxidative glucose metabolism, which is associated with higher levels of glucose storage in muscle and has been related to better insulin sensitivity [23]. These findings are in agreement with preclinical data showing that the overexpression of $C P T 1 b$ in skeletal muscle induces an increase in glycogen content [24].

Our present data also suggest that sirtuin proteins and, more particularly, SIRT1 and SIRT6 could be involved in the overexpression of CPTIb during ageing. Although SIRT1 expression did not significantly differ between our two groups, SIRT6 and SIRT2 expression was lower in our robust subjects. In line with this observation, SIRT2 expression was higher in insulin-resistant skeletal muscle cells, whereas inhibition of SIRT2 improved insulin sensitivity in a model in vitro [25]. The lower expression of SIRT6 in our robust subjects was also consistent with a previous preclinical study showing an increase of SIRT6 mRNA level with increasing age that can be reversed by exercise [26].

However, our research has a few limitations. First, frailty assessment in geriatric populations remains controversial. While the initial score as per Fried criteria is based only on physical aspects, other scores have since been further developed for multidimensional evaluations $[3,27]$. In fact, the present study used a new composite ageing indicator based 
equally on cognitive assessments and physical tests to determine our subgroups of frail and robust subjects. Unexpectedly, the frail subjects were older than the robust subjects, thereby requiring adjustment for age in further analyses. Nevertheless, such a discrepancy has been reported in previous studies based on other frailty scores, highlighting the difficulty of assessing frailty independently of age $[28,29]$. Furthermore, both of our groups displayed similar metabolic and anthropometric profiles at screening, including comparable levels of fasting glucose, fasting lipid, HbA1c and blood pressure. This suggests that our new ageing indicator is able to identify subjects at an early stage before the onset of significant metabolic changes [30]. Second, the number of subjects was relatively low in each of our groups. Yet, despite this, significant differences were demonstrated in both metabolic parameters and gene expression levels, although smaller differences, especially the level of SIRT1 expression, may not have been detected in our study. Finally, only gene expression was measured and, thus, there is the possibility that the increase in $C P T 1 b$ expression was not associated with an increase in levels of the protein itself. However, functional measurement of lipid oxidation by indirect calorimetry was in accordance with greater activity of $C P T 1 b$ in our robust subjects.

The present study represents a first step in our understanding of the factors involved in metabolic health with increasing age. The results are promising and need to be validated by a larger sample size; our findings also suggest that the expression of $C P T 1 b$ should now be assessed in longitudinal studies. Such studies could confirm that CPTIb expression increases with age to limit both intramyocellular lipid deposition and the development of insulin resistance. As previous preclinical studies have shown that physical activity 
stimulates $C P T 1 b$ expression [31,32], further work should also aim to determine whether the amount of physical activity is directly related to $C P T 1 b$ expression in older subjects. Moreover, it has been shown that physical training can improve muscle mitochrondrial quantity and oxidative capacity in older people [33]. In this case, a physical training programme might reverse the metabolic abnormalities associated with frailty at an earlier stage. Also, the putative effects of sirtuin histones on $C P T 1 b$ gene expression need to be explored in more detail in further experimental investigations.

In conclusion, our present study has demonstrated that robust subjects are more insulin-sensitive, oxidize more lipid in a fasting state and store more glucose during a euglycaemic-hyperinsulinaemic clamp test compared with frail subjects. The overexpression of $C P T 1 b$ in robust subjects could at least partly explain their better metabolic profile and might even limit the development of subsequent metabolic changes. In addition, our research may help to determine strategies to reverse frailty at an early stage and to promote metabolic health with increasing age.

\section{Conflicts of interest: none}

\section{Funding:}

The SU.VI.MAX 2 Study was funded by the French National Research Agency (grant number ANR-05-PNRA-010) and French Ministry of Health. C.B. was funded by the Fondation pour la Recherche Médicale (FRM; Foundation for Medical Research) during 
the writing of this article (grant number 40184). These funding bodies had no involvement in the design/conduct of the research, in data analysis/interpretation or in writing/approving the manuscript.

\section{Acknowledgments}

We are grateful to Aisling Burns for English proofreading.

\section{Appendix supplementary material}

Supplementary materials (Fig. S1, Tables S1-S3) associated with this article can be found at http://www.scincedirect.com at doi . . . 


\section{References}

[1] Aunan JR, Watson MM, Hagland HR, Søreide K. Molecular and biological hallmarks of ageing. Br J Surg 2016;103:e29-46. doi:10.1002/bjs.10053.

[2] Ng TP, Feng L, Nyunt MSZ, Feng L, Niti M, Tan BY, et al. Nutritional, Physical, Cognitive, and Combination Interventions and Frailty Reversal Among Older Adults: A Randomized Controlled Trial. Am J Med 2015;128:1225-1236.e1. doi:10.1016/j.amjmed.2015.06.017.

[3] Fried LP, Tangen CM, Walston J, Newman AB, Hirsch C, Gottdiener J, et al. Frailty in older adults: evidence for a phenotype. J Gerontol A Biol Sci Med Sci 2001;56:M146-156.

[4] Barbieri M, Gambardella A, Paolisso G, Varricchio M. Metabolic aspects of the extreme longevity. Exp Gerontol 2008;43:74-8. doi:10.1016/j.exger.2007.06.003.

[5] Barzilai N, Huffman DM, Muzumdar RH, Bartke A. The Critical Role of Metabolic Pathways in Aging. Diabetes 2012;61:1315-22. doi:10.2337/db11-1300.

[6] Johannsen DL, Conley KE, Bajpeyi S, Punyanitya M, Gallagher D, Zhang Z, et al. Ectopic Lipid Accumulation and Reduced Glucose Tolerance in Elderly Adults Are Accompanied by Altered Skeletal Muscle Mitochondrial Activity. J Clin Endocrinol Metab 2012;97:242-50. doi:10.1210/jc.2011-1798.

[7] Crane JD, Devries MC, Safdar A, Hamadeh MJ, Tarnopolsky MA. The Effect of Aging on Human Skeletal Muscle Mitochondrial and Intramyocellular Lipid Ultrastructure. J Gerontol A Biol Sci Med Sci 2010;65A:119-28. doi:10.1093/gerona/glp179.

[8] Hercberg S, Galan P, Preziosi P, Bertrais S, Mennen L, Malvy D, et al. The 
SU.VI.MAX Study: a randomized, placebo-controlled trial of the health effects of antioxidant vitamins and minerals. Arch Intern Med 2004;164:2335-42. doi:10.1001/archinte.164.21.2335.

[9] Kesse-Guyot E, Amieva H, Castetbon K, Henegar A, Ferry M, Jeandel C, et al. Adherence to nutritional recommendations and subsequent cognitive performance: findings from the prospective Supplementation with Antioxidant Vitamins and Minerals 2 (SU.VI.MAX 2) study. Am J Clin Nutr 2011;93:200-10. doi:10.3945/ajcn.2010.29761.

[10] Radloff LS. The CES-D Scale: A Self-Report Depression Scale for Research in the General Population. Appl Psychol Meas 1977;1:385-401. doi:10.1177/014662167700100306.

[11] McNair D, Kahn R. Self-assessment of cognitive deficits. Assess Geriatr Psychopharmacol. MPA; 1983.

[12] Laville M, Auboeuf D, Khalfallah Y, Vega N, Riou JP, Vidal H. Acute regulation by insulin of phosphatidylinositol-3-kinase, Rad, Glut 4, and lipoprotein lipase mRNA levels in human muscle. J Clin Invest 1996;98:43-9.

[13] Ferrannini E. The theoretical bases of indirect calorimetry: a review. Metabolism $1988 ; 37: 287-301$.

[14] Alligier M, Meugnier E, Debard C, Lambert-Porcheron S, Chanseaume E, Sothier M, et al. Subcutaneous adipose tissue remodeling during the initial phase of weight gain induced by overfeeding in humans. J Clin Endocrinol Metab 2012;97:E183-92. doi:10.1210/jc.2011-2314.

[15] Krishnan RK, Evans WJ, Kirwan JP. Impaired substrate oxidation in healthy elderly 
men after eccentric exercise. J Appl Physiol 2003;94:716-23. doi:10.1152/japplphysiol.00746.2002.

[16] Levadoux E, Morio B, Montaurier C, Puissant V, Boirie Y, Fellmann N, et al. Reduced whole-body fat oxidation in women and in the elderly. Int J Obes Relat Metab Disord J Int Assoc Study Obes 2001;25:39-44.

[17] Yu J, Auwerx J. The Role of Sirtuins in the Control of Metabolic Homeostasis. Ann N Y Acad Sci 2009;1173:E10-9. doi:10.1111/j.1749-6632.2009.04952.x.

[18] Royston JP. Algorithm AS 181: The W Test for Normality. J R Stat Soc Ser C Appl Stat 1982;31:176-80. doi:10.2307/2347986.

[19] RStudio Team. RStudio: Integrated Development Environment for R. Boston, MA: RStudio, Inc.; 2016.

[20] Bruce CR, Hoy AJ, Turner N, Watt MJ, Allen TL, Carpenter K, et al. Overexpression of carnitine palmitoyltransferase-1 in skeletal muscle is sufficient to enhance fatty acid oxidation and improve high-fat diet-induced insulin resistance. Diabetes 2009;58:550-8. doi:10.2337/db08-1078.

[21] Henique C, Mansouri A, Fumey G, Lenoir V, Girard J, Bouillaud F, et al. Increased mitochondrial fatty acid oxidation is sufficient to protect skeletal muscle cells from palmitate-induced apoptosis. J Biol Chem 2010;285:36818-27. doi:10.1074/jbc.M110.170431.

[22] Chee C, Shannon CE, Burns A, Selby AL, Wilkinson D, Smith K, et al. The relative contribution of intramyocellular lipid to whole body fat oxidation is reduced with age, but subsarcolemmal lipid accumulation and insulin resistance are only associated with 
overweight individuals. Diabetes 2016. doi:10.2337/db15-1383.

[23] Vaag A, Henriksen JE, Beck-Nielsen H. Decreased insulin activation of glycogen synthase in skeletal muscles in young nonobese Caucasian first-degree relatives of patients with non-insulin-dependent diabetes mellitus. J Clin Invest 1992;89:782-8. doi:10.1172/JCI115656.

[24] Hénique C, Mansouri A, Vavrova E, Lenoir V, Ferry A, Esnous C, et al. Increasing mitochondrial muscle fatty acid oxidation induces skeletal muscle remodeling toward an oxidative phenotype. FASEB J Off Publ Fed Am Soc Exp Biol 2015. doi:10.1096/fj.14257717.

[25] Arora A, Dey CS. SIRT2 negatively regulates insulin resistance in C2C12 skeletal muscle cells. Biochim Biophys Acta 2014;1842:1372-8. doi:10.1016/j.bbadis.2014.04.027. [26] Koltai E, Szabo Z, Atalay M, Boldogh I, Naito H, Goto S, et al. Exercise alters SIRT1, SIRT6, NAD and NAMPT levels in skeletal muscle of aged rats. Mech Ageing Dev 2010;131:21-8. doi:10.1016/j.mad.2009.11.002.

[27] Clegg A, Rogers L, Young J. Diagnostic test accuracy of simple instruments for identifying frailty in community-dwelling older people: a systematic review. Age Ageing 2014:afu157. doi:10.1093/ageing/afu157.

[28] Watanabe Y, Hirano H, Arai H, Morishita S, Ohara Y, Edahiro A, et al. Relationship Between Frailty and Oral Function in Community-Dwelling Elderly Adults. J Am Geriatr Soc 2016. doi:10.1111/jgs.14355.

[29] Lin P-S, Hsieh C-C, Cheng H-S, Tseng T-J, Su S-C. Association between Physical Fitness and Successful Aging in Taiwanese Older Adults. PLOS ONE 2016;11:e0150389. 
doi:10.1371/journal.pone.0150389.

[30] Cederholm T, Bosaeus I, Barazzoni R, Bauer J, Van Gossum A, Klek S, et al. Diagnostic criteria for malnutrition - An ESPEN Consensus Statement. Clin Nutr Edinb Scotl 2015;34:335-40. doi:10.1016/j.clnu.2015.03.001.

[31] Morifuji M, Sanbongi C, Sugiura K. Dietary soya protein intake and exercise training have an additive effect on skeletal muscle fatty acid oxidation enzyme activities and mRNA levels in rats. Br J Nutr 2006;96:469-75.

[32] Niu Y, Yuan H, Fu L. Aerobic exercise's reversal of insulin resistance by activating AMPK $\alpha$-ACC-CPT1 signaling in the skeletal muscle of C57BL/6 mice. Int J Sport Nutr Exerc Metab 2010;20:370-80.

[33] Irving BA, Lanza IR, Henderson GC, Rao RR, Spiegelman BM, Nair KS. Combined training enhances skeletal muscle mitochondrial oxidative capacity independent of age. J Clin Endocrinol Metab 2015;100:1654-63. doi:10.1210/jc.2014-3081. 


\section{Legends to figures}

Fig. 1. Flow chart of participant inclusion in the study.

Fig. 2. Skeletal muscle expression of eight genes involved in skeletal muscle metabolismcarnitine palmitoyltransferase $1 \mathrm{~b}(C P T 1 B)$ and 2 (CPT2), acetyl-coenzyme A (CoA) acyltransferase 2 (ACAA2), acetyl-CoA carboxylase beta (ACACB), malonyl-CoA decarboxylase $(M L Y C D)$, glycogen synthase 1 (GYS1), key regulator of lipid oxidation peroxisome proliferator-activated receptor-gamma coactivator 1-alpha (PPARGC1A) and pyruvate dehydrogenase kinase $4(P D K 4)$-in frail $(n=7)$ and robust $(n=8)$ subjects. mRNA levels (means \pm SEM) were normalized against expression of hypoxanthine phosphoribosyltransferase 1 (HPRT1). $* P<0.05$.

Fig. 3. Skeletal muscle expression of sirtuin $(S I R T)$ genes in frail $(n=7)$ and robust $(n=8)$ subjects. mRNA levels (means \pm SEM) were normalized against expression of hypoxanthine phosphoribosyltransferase 1 (HPRT1). SIRT4 expression was undetectable. * $P<0.05 ; * * P<0.01$.

Fig. 4. Scatterplots illustrating the relationship between carnitine palmitoyltransferase $1 \mathrm{~b}$ (CPT1B) and SIRT1, SIRT2, SIRT3, SIRT5, SIRT6 and SIRT7 expression, according to Spearman's correlation. Rho coefficients [bootstrap 95\% confidence intervals] are also indicated. mRNA: messenger RNA; HPRT1: hypoxanthine phosphoribosyltransferase 1. 


\section{Supplementary figure legend}

Fig. S1. Box plots showing a single outlier each (arrow) in SIRT1, SIRT2 and SIRT3 messenger RNA (mRNA) levels from one frail subject who was subsequently excluded from further mRNA analyses. HPRT1: hypoxanthine phosphoribosyltransferase 1 . 
Subjects from the SU.VI.MAX study

$$
\mathrm{N}=12741
$$

$\downarrow$

\section{Subjects from the SU.VI.MAX 2 study \\ $\mathrm{N}=6580$}

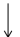

Assessment of aging indicator

-Did not meet inclusion criteria, $n=6$ -Declined to participate, $n=21$

- Other reasons, $n=4$
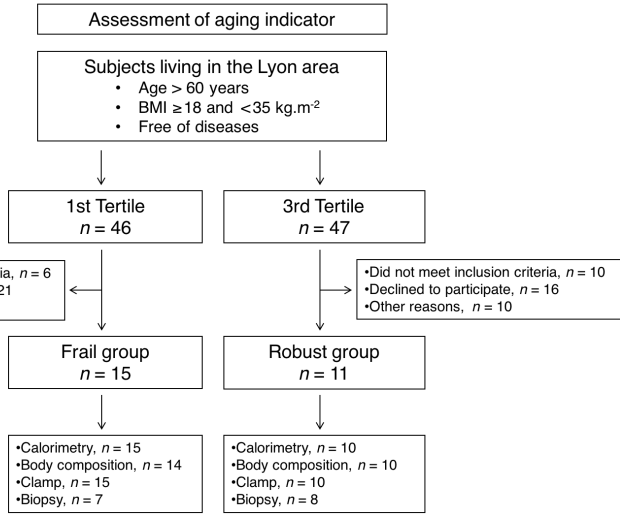
ACAA2/HPRT1

mRNA relative levels

mRNA relative levels
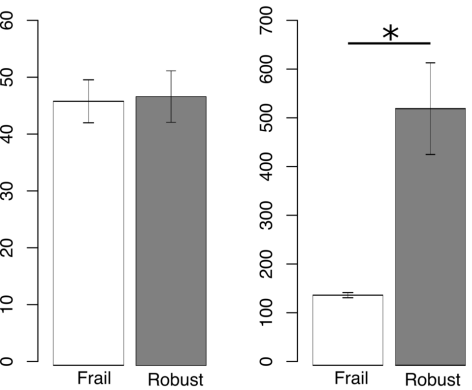

ACACB/HPRT1

mRNA relative levels

MLYCD/HPRT1

mRNA relative levels

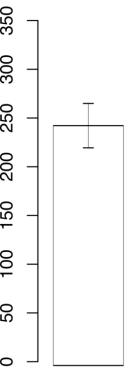

Frail

Robust

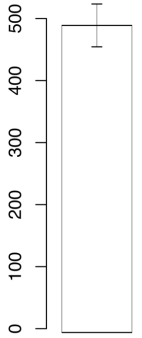

Frail
GYS1/HPRT1

mRNA relative levels

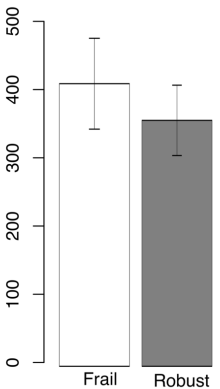

Frail

Robust

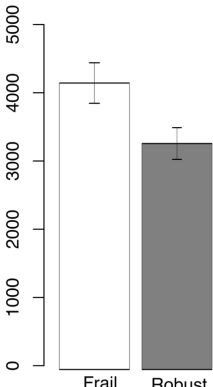

PDK4/HPRT1

mRNA relative levels

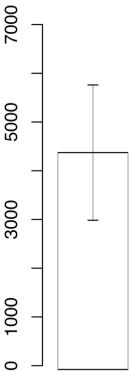

Frail 


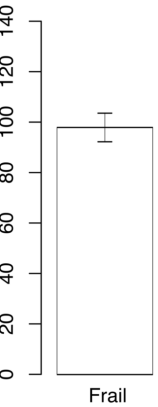

SIRT5/HPRT1

mRNA relative levels

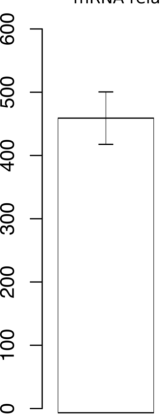

Frail

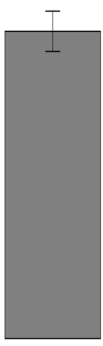

Robust

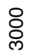

*

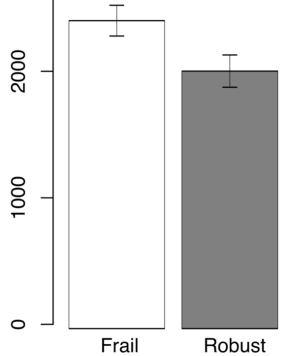

SIRT6/HPRT1

mRNA relative levels

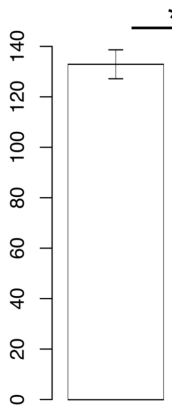

Frail $\star \star$

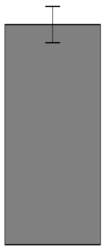

Robust
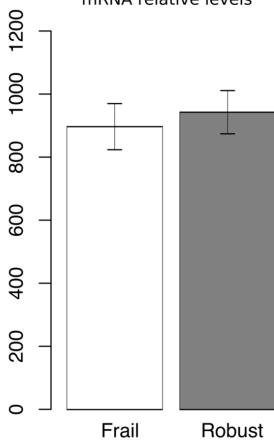

SIRT7/HPRT1

mRNA relative levels

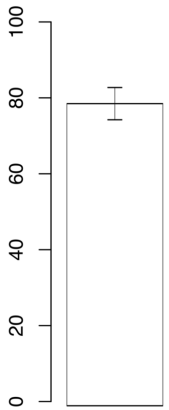

Frail

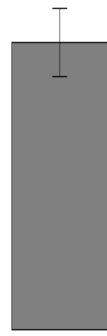

Robust 
Table I. Anthropometric and metabolic characteristics of frail and robust subjects at screening

\begin{tabular}{lccc} 
& Frail $(\mathbf{n}=\mathbf{1 5})$ & Robust $(\mathbf{n}=\mathbf{1 1})$ & $\boldsymbol{P}$ \\
\hline Composite ageing indicator & $7.3 \pm 0.6$ & $17.9 \pm 0.6$ & $<0.001$ \\
Age, years & $69 \pm 1$ & $64 \pm 1$ & $<0.01$ \\
Gender (male/female, $\mathrm{n} / \mathrm{n})$ & $8 / 7$ & $6 / 5$ & 1.00 \\
Weight, kg & $66.1 \pm 2.2$ & $69.3 \pm 3.1$ & 0.41 \\
Body mass index, kg/m ${ }^{2}$ & $24.1 \pm 0.6$ & $25.1 \pm 1.1$ & 0.39 \\
Waist size, cm & $85.3 \pm 2.2$ & $85.7 \pm 3.3$ & 0.92 \\
Mean blood pressure, mmHg & $98 \pm 2$ & $101 \pm 2$ & 0.23 \\
Fasting blood glucose, $\mu$ mol/L & $5.2 \pm 0.1$ & $5.3 \pm 0.2$ & 0.59 \\
HbA1c, $\%$ & $5.6 \pm 0.1$ & $5.7 \pm 0.1$ & 0.30 \\
Total cholesterol, mmol/L & $5.8 \pm 0.2$ & $6.1 \pm 0.3$ & 0.54 \\
HDL cholesterol, mmol/L & $1.6 \pm 0.1$ & $1.7 \pm 0.1$ & 0.48 \\
LDL cholesterol, mmol/L & $3.7 \pm 0.2$ & $3.9 \pm 0.2$ & 0.64 \\
Triacylglycerol, mmol/L & $1.1 \pm 0.1$ & $1.1 \pm 0.1$ & 0.60 \\
Values are means \pm SEM unless otherwise indicated; & & \\
HDL/LDL: high-density/low-density lipoprotein & & & \\
& & & \\
\hline
\end{tabular}


Table II. Resting energy expenditure (REE) and whole-body substrate oxidation in frail and robust subjects as assessed by calorimetry before (basal) and during euglycaemichyperinsulinaemic clamp tests

Frail $(\mathbf{n}=15) \quad$ Robust $(\mathbf{n}=10) \quad P$

\begin{tabular}{llll}
\hline REE, kcal & & & \\
Basal & $1282 \pm 42$ & $1355 \pm 49$ & 0.52 \\
Clamp & $1348 \pm 42$ & $1396 \pm 55$ & 0.71
\end{tabular}

Glucose oxidation, $\mathrm{mg} \cdot \mathrm{kg}^{-1} \cdot \mathrm{min}^{-1}$

$\begin{array}{llll}\text { Basal } & 1.08 \pm 0.13 & 0.67 \pm 0.13 & 0.21 \\ \text { Clamp } & 2.63 \pm 0.12 & 2.20 \pm 0.24 & 0.58\end{array}$

Non-oxidative glucose metabolism, mg.kg ${ }^{-1} \cdot \min ^{-1}$
Clamp
$4.78 \pm 0.51$
$5.58 \pm 0.64$
$<0.01$

Lipid oxidation, $\mathrm{mg} \cdot \mathrm{kg}^{-1} \cdot \mathrm{min}^{-1}$
Basal
$0.70 \pm 0.06$
$0.94 \pm 0.08$
$<0.05$
Clamp
$0.11 \pm 0.04$
$0.29 \pm 0.06$
0.29

Respiratory quotient, $\mathrm{VCO}_{2} / \mathrm{VO}_{2}$

$\begin{array}{llll}\text { Basal } & 0.82 \pm 0.01 & 0.77 \pm 0.01 & 0.069 \\ \text { Clamp } & 0.97 \pm 0.01 & 0.93 \pm 0.02 & 0.20\end{array}$

Values are means \pm SEM 